\title{
Large Unresponsive Zones Appear in Cat Somatosensory Cortex Immediately After Ulnar Nerve Cut
}

\author{
Cheng-Xiang Li, Robert S. Waters, Akinniran Oladehin, Eldridge F. Johnson, \\ Carl A. McCandlish and Robert W. Dykes
}

\begin{abstract}
The organization of the primary somatosensory cortex innervated by the ulnar nerve was studied before and immediately after ulnar nerve transection in 11 cats electrophysiologically mapped under Nembutal or Ketamine anesthesia. The cortex was reexamined a second time beginning $42 \mathrm{hr}$ after nerve transection in four cats anesthetized with Nembutal. One additional sham-operated control was also mapped. The region of cortex formerly served by the ulnar nerve remained largely unresponsive to somatic stimulation independent of the type of anesthetic used during recording. Nonetheless, animals anesthetized with Ketamine had more new responsive sites in deafferented cortex following nerve cut than cats anesthetized with Nembutal. New responses, when observed, were evoked by stimulation of a region of skin adjacent to the region served by the ulnar nerve. These findings suggest that the immediate response to deafferentation of somatosensory cortex is a limited acquisition of novel responses restricted to a region immediately adjacent to cortex containing normal afferent input.
\end{abstract}

\begin{abstract}
Résumé: Zones étendues de non-réponse apparaissant dans le cortex somesthésique du chat immédiatement après la section du nerf cubital. Nous avons étudié l'organisation du cortex somesthésique primaire innervé par le nerf cubital avant et immédiatement après la section du nerf cubital chez 11 chats cartographiés au point de vue électrophysiologique sous anesthésie au Nembutal ou à la kétamine. Le cortex a été réaxaminé une seconde fois 42 heures après la section nerveuse chez 4 chats anesthésiés au Nembutal. Un chat contrôle ayant subi une opération factice a aussi été cartographié. La région du cortex desservie antérieurement par le nerf cubital est demeurée en grande partie insensible à la stimulation somatique, quel qu'ait été le type d'anesthésie utilisé pendant l'enregistrement. Cependant, les animaux anesthésiés à la kétamine avaient plus de sites sensibles nouveaux dans le cortex désafférentié suite à la section nerveuse que les chats anesthésiés au Nembutal. Quand de nouvelles réponses étaient observées, elles étaient provoquées par la stimulation d'une région de la peau adjacente à la région innervée par le nerf cubital. Ces observations suggèrent que la réponse immédiate à la désafférentiation du cortex somesthésique est une acquisition limitée de réponses nouvelles, restreintes à la région immédiatement adjacente au cortex contenant l'infux afférent normal.
\end{abstract}

Can. J. Neurol. Sci. 1994; 21: 233-247

The details of the body representation in primary somatosensory cortex have been studied ${ }^{1-3}$ extensively since the introduction of multiunit mapping techniques. ${ }^{4}$ The notion that loss of sensory input to the map would leave a permanent vacancy was brought into question by three independent groups of investigators. ${ }^{5-7}$ Their findings suggested that loss of sensory input to a body part, by deafferentation, did not produce an empty region in the cortical map at the location formerly served by the deafferented body part; rather, neurons in the newly deprived region responded to stimulation of neighboring regions of the body. These early studies were performed in cats anesthetized with either an inhalation or a barbiturate anesthetic. Deafferentations involved peripheral nerve transection, ${ }^{5}$ dorsal root transection ${ }^{6}$ or reversible epidural blocks using a local anesthetic. ${ }^{7}$ Following deafferentation, animals were examined at different times, ranging from immediately after the nerve injury ${ }^{7}$ to several weeks later ${ }^{5.6}$ In each case the cortex formerly served by sensory input from the deafferented limb was shown to respond to new input from a neighboring region of the body.

These early studies in cat were extended by Merzenich, Kaas and their colleagues ${ }^{8-10}$ who documented the phenomenon of cortical reorganization in several non-human primates and

From the Departments of Anatomy and Neurobiology, (C.-X.L., R.S.W., E.F.J., C.A.M.) and Physical and Occupational Therapy (A.O.). University of Tennessee, Memphis, College of Medicine, Memphis; and the Department of Physiology, University of Montreal, Montreal (R.W.D.).

RECEIVED AUGUST 10, 1992. ACCEPTED IN FINAL FORM MARCH 7. 1994.

Reprint requests: Robert S. Waters, Department of Anatomy and Neurobiology, University of Tennessee, Memphis, 875 Monroe Avenue, Memphis, Tennessee, USA 38163 
described in detail the temporal sequence of reorganization of the hand representation within monkey primary somatosensory cortex following deafferentation. ${ }^{9}$ While this work was important in elucidating the dynamic process of reorganization, it also raised important questions concerning the underlying somatosensory cortical microstructure necessary to account for the phenomenon.

The phenomenon of cortical reorganization is now welldocumented in monkeys, ${ }^{8-11}$ raccoon, ${ }^{12-15}$ bat, ${ }^{16,17}$ and rat. ${ }^{18,19}$ Despite the fact that the first reports concerning cortical reorganization were made in cats some time ago, cortical function immediately following deafferentation has not been described in detail nor has the sequence of changes that follow deafferentation been documented in this species. Therefore, we set out to study the somatosensory representation of the forepaw and digits in normal cats and the possible changes that occur in this representation immediately after ulnar nerve transection. To acquire this information without confounding variability among different individuals with cortical reorganization, each animal was studied before and immediately after nerve transection and several animals were further studied at selected times beginning 42 hours later. Our results suggest that at all times tested following deafferentation the majority of neurons recorded in the region of cortex served exclusively by the ulnar nerve were only mildly responsive to cutaneous stimulation. This finding was independent of whether animals were examined under Nembutal or Ketamine anesthesia. Those few sites that were responsive in the ulnar nerve innervated cortical zone immediately after deafferentation were drivable, in large part, by stimulation of skin regions of the paw lying immediately adjacent to the ulnar nerve innervated sites or by high threshold tap stimulation.

Multiple mapping was also conducted in a sham-operated control animal to assess whether possible damage from multiple penetrations might produce a cortex mildly responsive to cutaneous stimulation. In addition, the sham-operated control animal also provided information as to whether cortical neurons are constantly undergoing changes in their receptive fields; if this were the case, such changes could be mistaken for cortical reorganization in deafferented animals.

\section{MethodS}

\section{General Preparation}

Experiments were carried out on 11 adult cats. Nine were anesthetized with an interperitoneal injection of Nembutal (35 $\mathrm{mg} / \mathrm{kg}$, i.p.), and two were injected with Ketamine $(100 \mathrm{mg} / \mathrm{kg}$, i.m.) and prepared for surgery. All animals were given supplemental doses of anesthetic ( $10 \%$ of the original dose) at regular hourly intervals or whenever necessary to maintain areflexia. The animal was placed on a heating pad and the body temperature was maintained between 37 and $38.5^{\circ} \mathrm{C}$. Under surgical anesthesia, the hair on the left forearm and shoulder, left hindlimb, right thigh, and crown of the skull was shaved. The femoral vein was cannulated and used to infuse a solution of $5 \%$ dextrose and periodically to deliver supplemental doses of anesthetic. The trachea was cannulated. The left forearm was placed into a custom-made hand holder which was secured to the recording table. The holder was constructed from aluminum tubing and fashioned into a rectangularly shaped loop. The paw was suspended by sutures threaded through the ends of the claws. A midsagittal cut was made in the skin to expose the skull, the skin was reflected, and a craniotomy was performed over the sigmoid gyrus. The dura was removed and the cortical surface was covered with warmed silicon fluid. A high resolution photograph was taken of the cortical surface and enlarged. It served to record the locations of electrode penetrations.

\section{Electrophysiological Recording and Mapping}

A carbon-fiber electrode, ${ }^{20}$ attached to a Narishige microdrive, was used to record multiunit responses from neurons in the somatosensory cortex. The electrode penetration sites were separated by $250-\mu \mathrm{m}$ steps. Since experiments were designed to map a well-defined area, recording sites were confined to the crown of the post sigmoid gyrus. The mapped region was bordered laterally by the medial bank of the coronal sulcus, anteriorly by a region in front of the representation of the third digit which borders on cortex responsive to high-threshold stimulation (tap, joint movement), posteriorly by a region responsive to cutaneous stimulation of the forearm and wrist, and medially by another high-threshold region. All recordings were made at a depth of 800 microns below the cortical surface. This depth was selected to increase the level of reliability in returning the electrode to the previous recording site during the subsequent mapping. Our previous mapping studies in cat have shown that a depth between 750 and $850 \mu \mathrm{m}$ generally yields the strongest responses to peripheral stimulation. ${ }^{21}$ All cortical areas were mapped by the same investigator before and after nerve cut, and the receptive field was corroborated by at least one additional investigator. Following nerve cut, all animals were immediately remapped and in 4 cases the cortex was mapped an additional time $42 \mathrm{hr}$ after nerve cut. All animals were continuously maintained under a surgical dose of anesthesia throughout all surgical preparation and mapping procedures, and animals did not recover from the surgical anesthesia.

After the recording session, all animals received an injection of Nembutal $(20 \mathrm{mg} / \mathrm{kg}$ ); electrolytic lesions were then placed at selected sites in the cortex. The animal was then given an overdose of Nembutal $(50 \mathrm{mg} / \mathrm{kg}$ ) and perfused with saline followed by buffered formalin. Brains were removed from the skull, stored in a $10 \%$ formalin solution with $20 \%$ sucrose for several days and then cut at $60-\mu \mathrm{m}$ thickness on a freezing microtome. Sections containing electrolytic lesions were reconstructed with the aid of a drawing tube attached to a light microscope or an Aus Jena microprojector.

\section{Peripheral Deafferentation}

In all cases, the ulnar nerve was severed within $2 \mathrm{~cm}$ distal to the elbow. Before deafferentation, the nerve was identified by placing a cuff electrode around the nerve and recording evoked responses following stimulation of the forepaw. Additional testing procedures were sometimes utilized to further identify the nerve: these were mechanical pressure (lightly pinching the nerve with forceps and observing the muscle contraction) or stimulation through the cuff electrodes with constant current (100 $\mu \mathrm{A}$ or less) and observing the subsequent contraction.

\section{Receptive Field Reconstruction}

The receptive field of neuron(s) was delineated at each cortical site by stimulation of the forepaw skin with a fine wooden probe or deflection of the hairs with a camel-hair brush. The receptive field was then outlined on a standard drawing of the paw. The standard drawing was generated (Figure 1) by measuring 
the size and distances between prominent landmarks on the paw surface ${ }^{21}$ and subdividing it into three regions running distal (a) to proximal (c); each digit is divided into an ulnar and radial side, and the web (w) is given an individual identification. On the ventral side, the distal-proximal relationships continue, except that the region distal to the metacarpal pad (MP) contains only divisions $\mathbf{a}$ and $\mathbf{b}$. A representative pad on the ventral side is identified by the digit served and by the letter $\mathbf{p}$. The wrist $(\mathbf{W})$, like the forepaw, is divided into three sections $\mathbf{a}, \mathbf{b}, \mathbf{c}$.

\section{Forepaw Zones}

The innervation of the forepaw was described previously. ${ }^{21}$ It consists of zones innervated by a single peripheral nerve, autonomous zone, and overlap zones which are innervated by two or more nerves. ${ }^{21,24}$ While there exists a degree of variability between animals and even between left and right paws, in all cases only the ulnar nerve innervates the entire fifth digit (D5), digit pad, and the ulnar side of the web between D4-5. The autonomous zone of the ulnar nerve is shown in Figure 1 by stippling; it comprises the entire dorsal-ventral extent of D5 and a part of the carpal pad and continues into the wrist and forearm (not illustrated). The autonomous ulnar nerve zone is surrounded on both sides by overlap zones which receive input from the ulnar nerve in addition to input from either the median or the radial nerve or both. The overlap zone, between the ulnar and median nerves, in greater than $80 \%$ of the cases studied included the radial side of the web between D4-5, ulnar onethird of the metacarpal pad, the pad of D4, and the ulnar side of D4. ${ }^{21}$ The overlap zone for the ulnar and radial nerves included

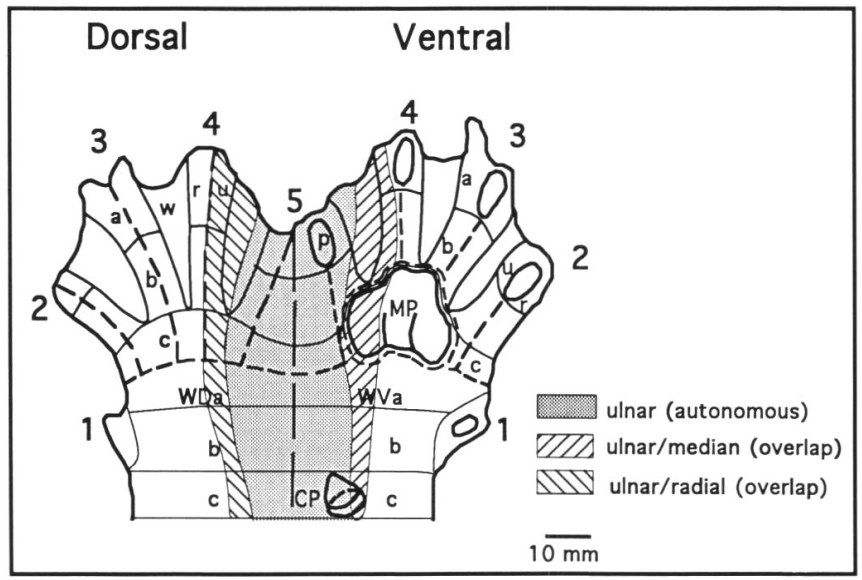

Figure 1. Line drawing of the standard cat paw showing the pattern of ulnar nerve innervation of the forepaw. The autonomous zone of skin is innervated (stippled) exclusively by the ulnar nerve, and the overlap zones are innervated by input by both the ulnar and median nerves and/or the ulnar and radial nerves (diagonal stripes). The forepaw map is joined together at the division between the dorsal and ventral surfaces on the ulnar side of the paw. The map was generated by measuring the distances of prominent landmarks on the paw surface of a perfused cat, removing the skin, flattening and stretching the skin to the previously measured distances, and tracing the resulting outline on graph paper. Paw and wrist are further divided into three sections lettered $a, b, c$. The ulnar $(\boldsymbol{u})$ and radial $(\boldsymbol{r})$ sides of the paw are indicated as are the web (w), metacarpal pad (MP), and carpal pad (CP). Dorsal and ventral sides of wrist are indicated as $W D$ and $W V$, respectively. Pad is indicated by $p$. the radial side of the web between D4-5 and the ulnar aspect of the dorsal part of D4.

\section{RESULTS}

The part of the primary somatosensory cortex served by the ulnar nerve as well as the cortex bordering the ulnar nerve representation was mapped in 11 animals. Subsequently, each animal underwent ulnar nerve transection and the cortex was remapped immediately following nerve cut. In 4 animals the cortex was remapped a second time 42 hours after nerve cut. One additional cat served as a sham-operated control. This report focuses on the immediate consequences of the nerve transection on the organization of the primary somatosensory cortex.

\section{General Organization of the Ulnar Representation in Somatosensory Cortex}

The location of cortex explored is shown in Figure 2. This region extends nearly $5 \mathrm{~mm}$ in the anterior-posterior direction and approximately $3 \mathrm{~mm}$ in the mediolateral direction encompassing an important part of the forelimb cutaneous representation in primary somatosensory cortex. Physiologically, this region receives input from digits 4 and 5 (D4, D5) and a portion of D3 (the remaining part of D3 as well as the D2 and D1 representations are located along the medial bank of the coronal sulcus) and extends into the wrist and forearm representations. On the anterior edge of the map, neurons which receive cutaneous input border neurons that are driven primarily by stimulation of muscles or by manipulation of joints. This anterior region serves to mark the border of the area studied and will not be described further.

The data sets for three representative cases are illustrated in Figure 3. Each site where an electrode penetration was made is shown by a filled circle (responsive site) or open circle (unresponsive site). The lower part of each panel immediately beneath the sulcus shows the general ordering of body part representation encountered in terms of forearm, wrist, and digits. At this level of reconstruction it is clear that the cortex is somatotopically organized with the representation of the

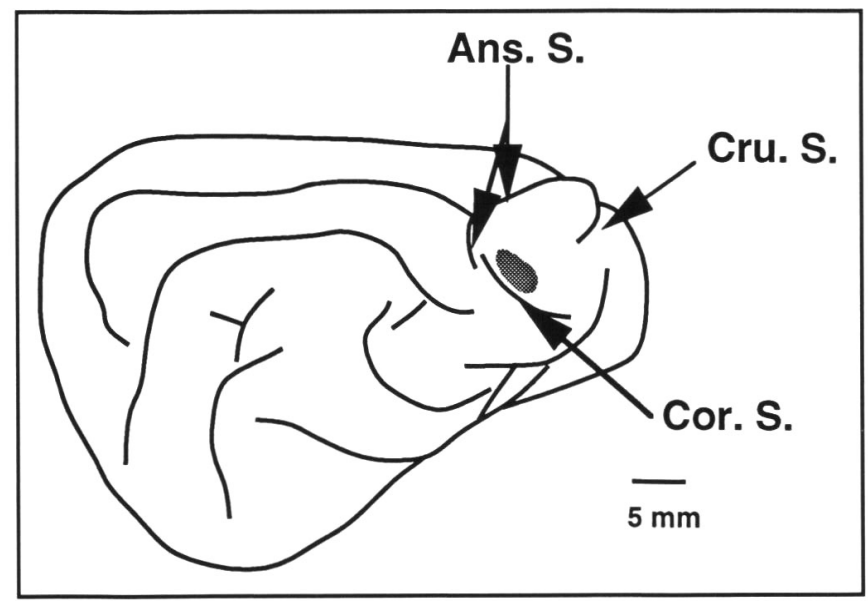

Figure 2. Drawing of the region studied (stippled) in cat primary somatosensory cortex. Ans. $S$. = Ansate Sulcus; Cru. $S .=$ Cruciate Sulcus; Cor. S. = Coronal Sulcus. 
forearm located most posterior, followed anteriorly by the representation of the wrist and digits, respectively. Note, in this figure, the partially shifted overlapping character of the representation of body parts is evident. Further, in some cases the same body part may be represented at two or more sites separated by the representation of a neighboring body part.

Another view of the organization of this region is obtained when the data are expressed in terms of the peripheral nerves which contribute to the activation of a particular cortical site (Figure 4). In this figure the locations of the receptive field(s) are coded at each recording site according to the regions defined in Figure 1. Two examples of receptive fields found at two of these sites are shown in the lower part of the figure. This region of cortex contained zones that received either exclusive input from the ulnar nerve (ulnar nerve zone) or input from the ulnar nerve in combination with the radial and/or median nerves

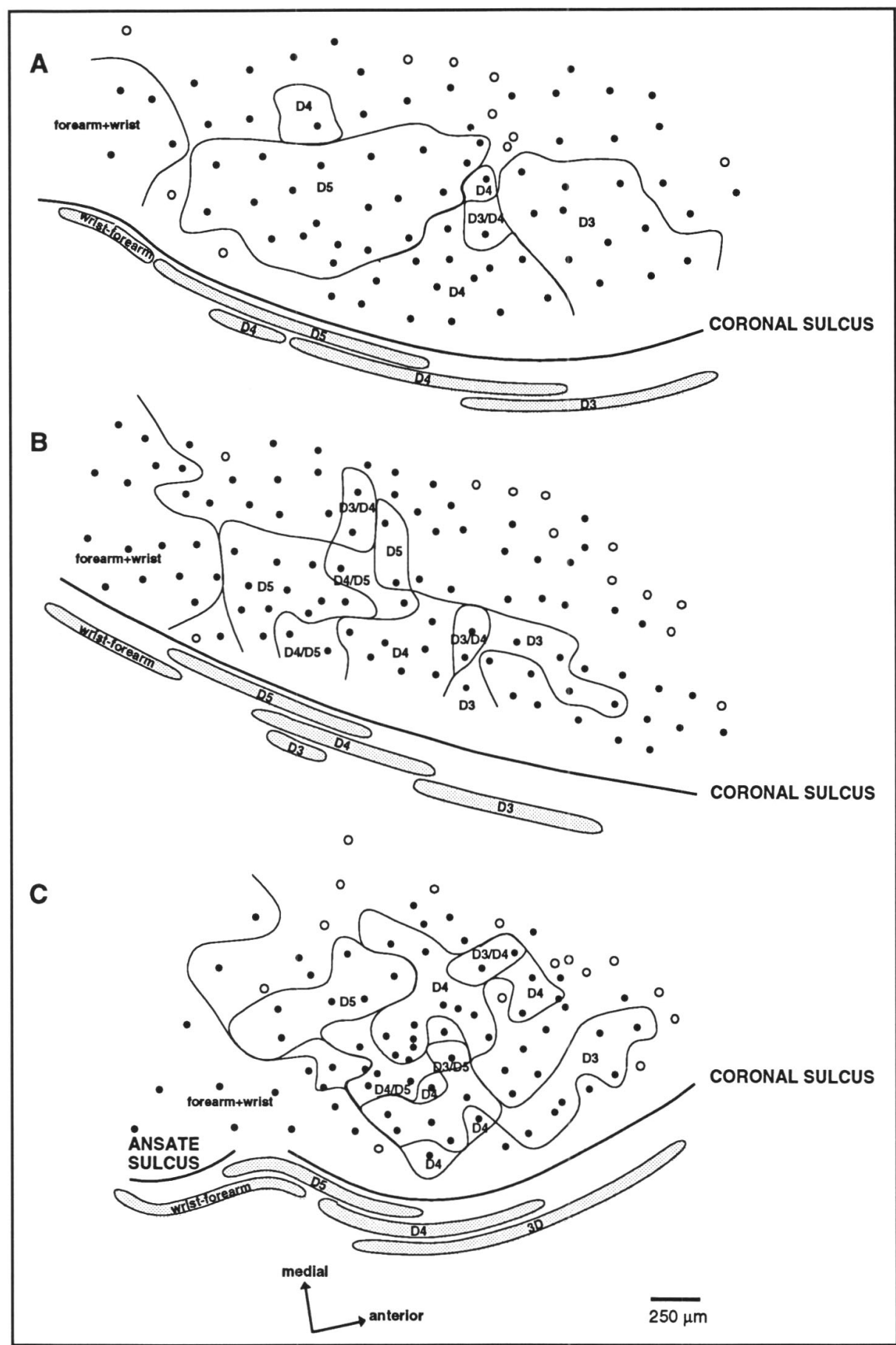

Figure $3 A, B, C$. Data sets from three animals illustrating the location of the forearm, wrist, and digit representation in the region studied in primary somatosensory cortex. The sausage-shaped figures below the Coronal Sulcus indicate the general somatotopic organization of this region. Note that only the representation illustrated in C extends to the Ansate Sulcus. Filled circle $=$ responsive site; open circle = unresponsive site. 
(combined nerve zone). These two zones are shaded differently in Figure 4; ulnar nerve zone cortex receiving exclusive input from the ulnar nerve is shown as dark stippling and combined nerve zone cortex receiving input from either the radial or median as well as the ulnar nerve is shown with light stippling. The ulnar nerve zone receiving exclusive input from the ulnar nerve includes the entire representation of D5 and a portion of the wrist and forearm on the ulnar side of the forearm. An example of a receptive field recorded from this zone is shown at the lower left. The receptive field recorded at this site was restricted to the ventral aspect of D5 including the pad, and a portion of the dorsal side of D5 coursing along the entire proximal-distal extent.

The illustration shown at lower right of Figure 4 is an example of a cortical neuron receiving input from the radial and/or median as well as the ulnar nerve within the combined nerve zone; the receptive field was restricted to the dorsal surface of D4 toward the ulnar side. This region of skin is innervated by both the ulnar and radial nerves, therefore, transection of the ulnar nerve alone will remove only partial input into this cortical region. The ulnar nerve overlaps with both the radial and median nerves to form two zones of combined innervation in the cortex. Within this combined nerve zone cortex there appears to be some reflection of this dual origin; the ulnar-radial overlap is located anteriorly, and the ulnar-median nerve overlap is located posteriorly. A further delineation of the somatotopy of the individual nerves contributing to the combined nerve zone will require more closely spaced electrode penetrations.

These two zones were seen in each animal; however, there were individual differences in the size and shape of these zones. Figure 5 illustrates this point in the same three animals shown in Figure 3 and serves to highlight individual differences in the size and shape of the two zones. In the example illustrated in Figure 5A, the zone receiving exclusive ulnar nerve input (dark stipple) is large compared to the size of the zone receiving combined nerve input (light stipple). The case illustrated in Figure $5 B$ shows the two zones to be nearly equal in size, while in the example in Figure 5C, the exclusive ulnar nerve input zone is smaller than the zone receiving combined nerve input.

With a $250-\mu \mathrm{m}$ electrode spacing, we observed a range of 5 to 17 cortical sites which could be included within the ulnar nerve zone. The combined nerve zone contained 13 to 19 sites

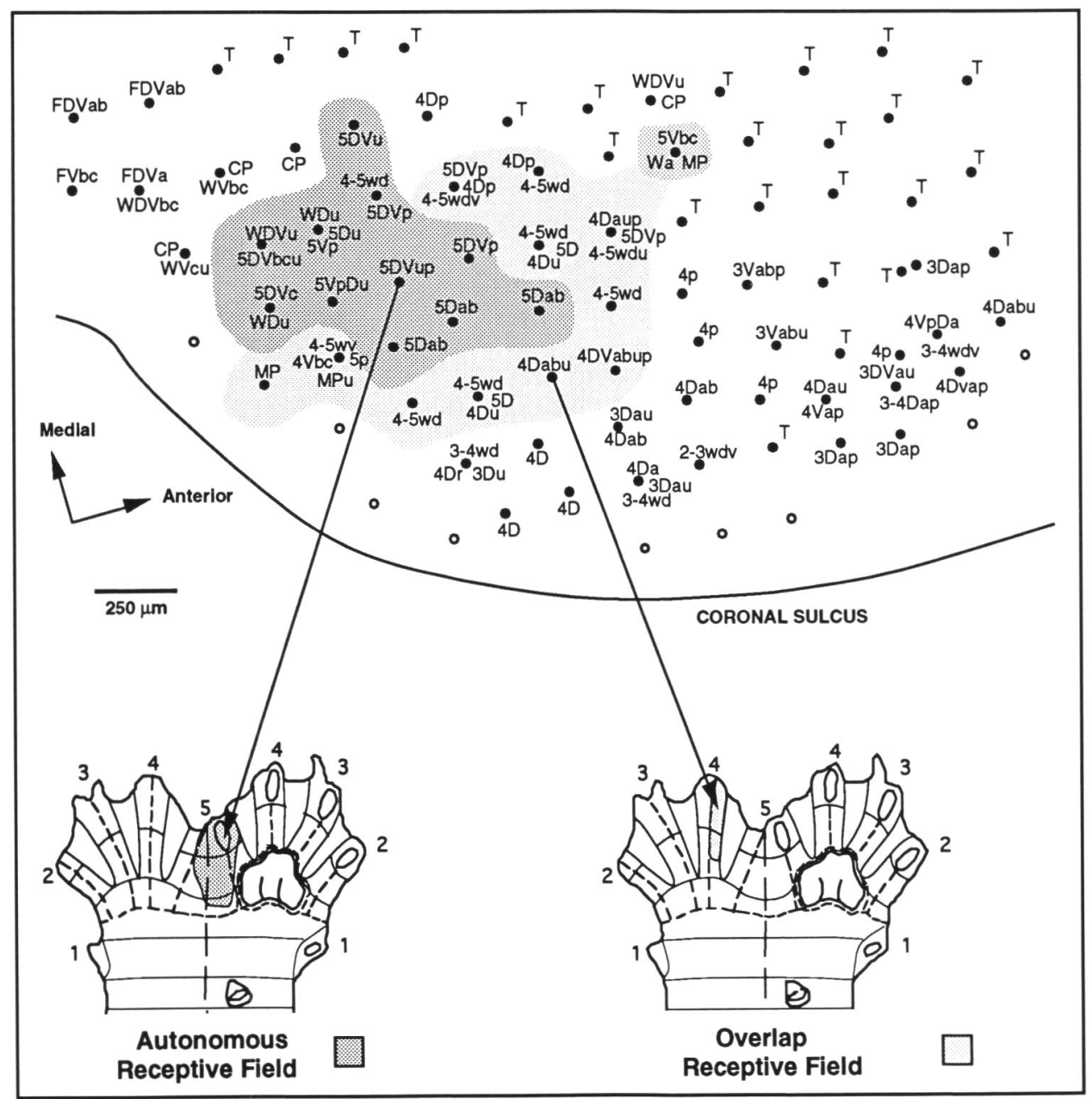

Figure 4. Reconstruction of recording sites showing the ulnar nerve zone (dark stipple) and combined nerve zone (light stipple) along with a representative example of receptive fields recorded in each zone. Electrode penetrations are indicated by circles (responsive sites indicated by filled circles, unresponsive sites indicated by open circles). Below are reconstructions of receptive fields recorded in each zone at the sites indicated by arrows. Numbers (top reconstruction) represent the digits, $F=$ forearm, $V=$ ventral, $D=$ dorsal, $p=$ pad, $M P=$ metacarpal pad, $C P=$ carpal pad, $W=$ wrist, $w$ (lower case $)=w e b, T=$ high threshold tap, $u=$ ulnar side, $r=$ radial, letters $a, b, c$ represent distal to proximal locations. 


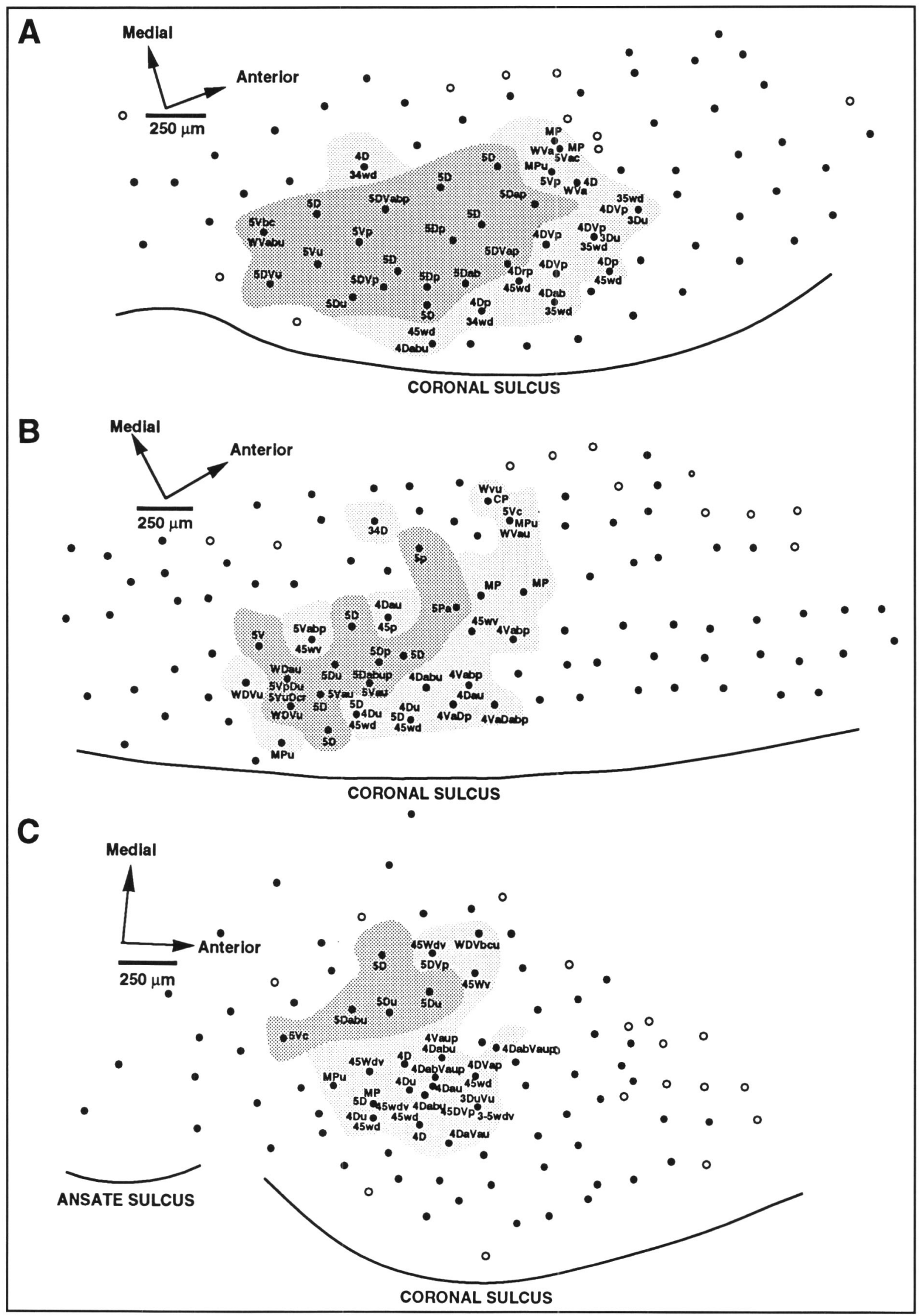

Figure 5. Reconstruction of ulnar nerve zone (dark stipple) and combined nerve zone (light stipple) for three cases. Note the variability in the size and shape of the zones in the three examples. A. Large ulnar nerve zone; B. Equivalent size ulnar nerve and combined nerve zones; C. Large combined nerve zone. 
in different animals when both the median and radial nerve contributions were included. From these data we can estimate that the ulnar nerve zone cortical area ranged from 1.25 to 4.25 $\mathrm{mm}^{2}$ and the combined nerve zone ranged from 3.25 to 4.75 $\mathrm{mm}^{2}$. Thus a large expanse of cortex is influenced by the ulnar nerve (e.g., 4.5 to $9.0 \mathrm{~mm}^{2}$ ).

Within normal somatosensory cortex, penetrations were occasionally unresponsive to cutaneous stimulation. There was no obvious relationship among these sites, and their locations were not consistent among animals. Thus it was difficult to elaborate hypotheses about the significance of these unresponsive sites.

\section{Immediate Consequences of Nerve Cut}

To explore the immediate consequences of nerve transection, the ulnar nerve was cut proximal to the elbow immediately following the completion of the original map, and first remapping (first remap) was started. Each recording site was remapped in the original sequence. The electrode was maneuvered over the previous recording site, as identified from a mark placed on a photograph of the surface. It was lowered again to a depth of $800 \mu \mathrm{m}$. Three examples of the results from this first remapping are illustrated in Figure 6. These serve to illustrate the variety of responses recorded immediately following nerve transection. The upper part of Figure 6 shows the map prior to nerve section with the ulnar nerve zone shaded and the combined nerve zone unshaded. The receptive field designation and location of recording sites are indicated. The lower part of Figure 6 shows the two zones but this time the receptive field data are replaced by a symbol indicating the relationship between the receptive field recorded following nerve cut and the original receptive field recorded at that site. Five categories of responses are illustrated. If a site was responsive to stimulation a determination was made as to whether the response was unchanged (cross), had a new receptive response (asterisk), had lost part, but not all of the original receptive field (triangle), or was driven only by high threshold tap (diamond). Unresponsive sites are indicated by open circles.

\section{Ulnar Nerve Zone Cortex}

Immediately following nerve cut, the majority of recording sites within the ulnar nerve zone were unresponsive even to high threshold tap stimulation. However, responses occurred at $20 \%$ of the sites. These were equally likely to be the result of high threshold tap stimulation or the appearance of a new lowthreshold receptive field. When a new low threshold response

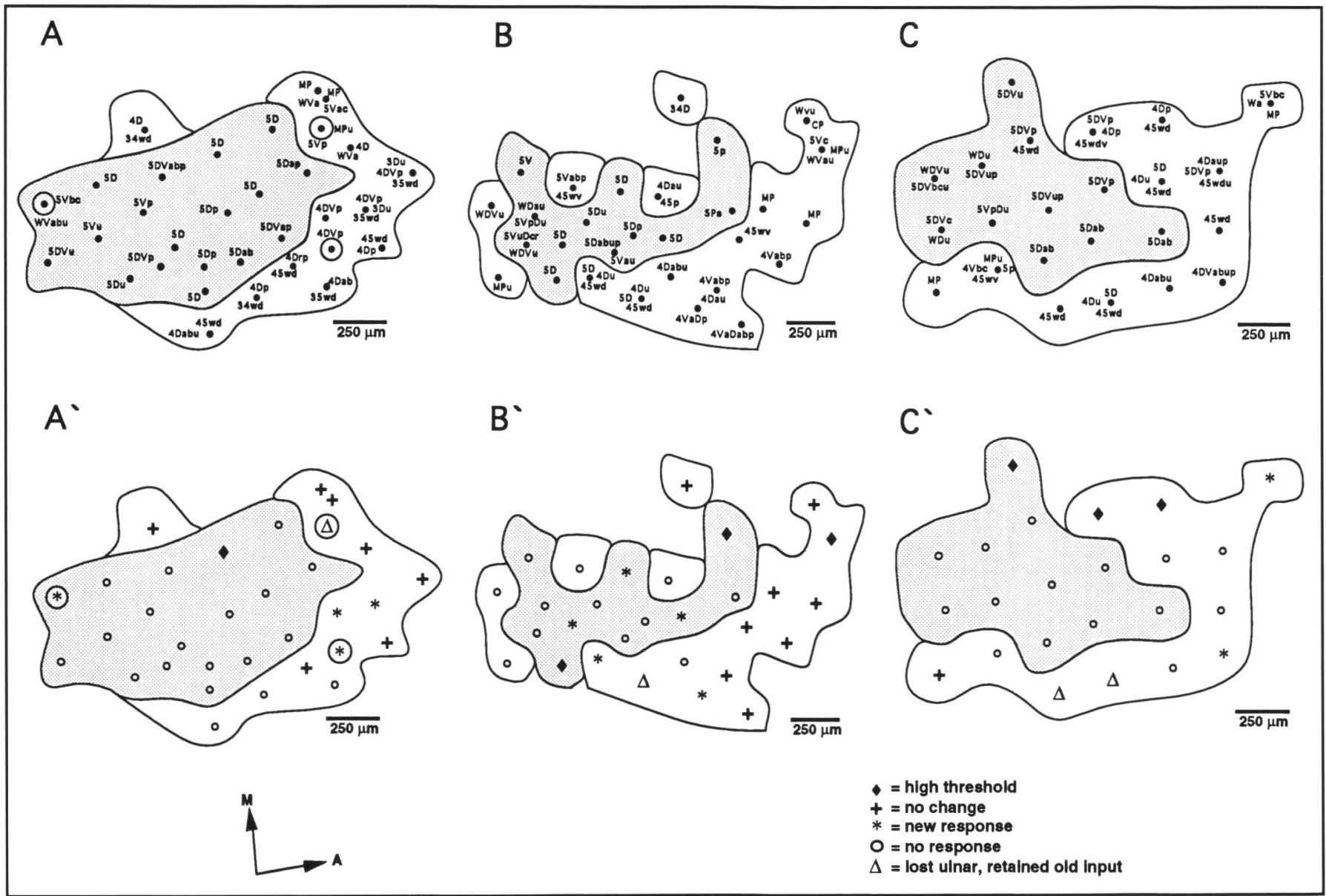

Figure 6. Reconstruction of ulnar nerve zone and combined nerve zone before (upper) and immediately after (lower) transection of the ulnar nerve for three cases. Recording sites in upper figures are indicated by black dots and receptive fields recorded at each site are indicated by the nomenclature introduced in Figure 1. Symbols in lower three figures indicate the receptive fields recorded at each site following nerve cut as compared to receptive fields recorded at the same sites before nerve cut. Large circles in A and A' show locations where receptive fields are reconstructed in Figure 7. 
was encountered, the cortical site was most likely driven from a region of skin adjacent to the previously active site. This new receptive field often was located, at least in part, within an overlap region of skin. An example of a cortical site where a new response was recorded following nerve cut is shown in Figure $6 A^{\prime}$. This site formerly responded to stimulation of the ventral side of D5 adjacent to the MP, but was not drivable by stimulation of MP. Following nerve cut, this site was no longer responsive to stimulation of D5, but could now be driven by stimulation of MP (this is illustrated in the paw maps shown in Figure 7A, $\left.A^{\prime}\right)$. Note the proximity of the new field to the previously recorded field. While only one new response was found in the example illustrated in Figure 6A', three appeared in the case shown in Figure 6B' following nerve cut. This was the most found in any of the 11 animals mapped. In contrast, the animal illustrated in Figure 6C showed no new responses following nerve cut. High threshold responses were recorded in each animal but these were difficult to localize because the intensity of stimulus required to drive the cortical neurons produced disturbances throughout the paw.

\section{Combined Nerve Zone Cortex}

Removal of the contribution by the ulnar nerve often resulted in changes in the combined nerve zone of the map that were not easily predictable. For example, it was not unusual for unresponsive sites to appear in the combined nerve zone. In fact, they were observed in nearly $45 \%$ of the sites and were present in every experimental animal studied. Responses with new receptive fields were also found in the combined nerve zone, and they represented an enlargement of the representation of the median or radial components. An example of the receptive field recorded in the combined nerve zone following nerve cut is shown in Figure 7B, B'. Note that the field shifted to the tip of D3 and extended onto the ventral side to the pad of D3. Several high-threshold sites were also observed in each animal. At some sites in the combined nerve zone the receptive field included a part of the skin served by the pure ulnar nerve. In many of these cases deafferentation removed the pure ulnar portion of the receptive field, leaving the remaining part of the field intact. These response types are indicated by triangles. The receptive field of cells recorded in the combined nerve zone where the ulnar component is lost after nerve cut is shown in Figure $7 \mathrm{C}$, $C^{\prime}$. In this case, the representation on the ventral side of D5 was lost, but the median component remained intact. Perhaps the most unexpected change was that more than $25 \%$ of the sites in the combined zone became unresponsive even to relatively intense mechanical stimuli. Figure 7 illustrates some of these receptive fields.

\section{Multiple Remapping}

The cortex was remapped a second time (second remap) beginning at 42 hours following nerve cut in four animals. Two of these cats, previously described in Figure 6 ( $A$ and $C$ ) will be used in Figure 8 to illustrate our findings. The upper half of Figure 8 shows the results of the first remap following nerve cut and is identical to that previously illustrated. The lower half of

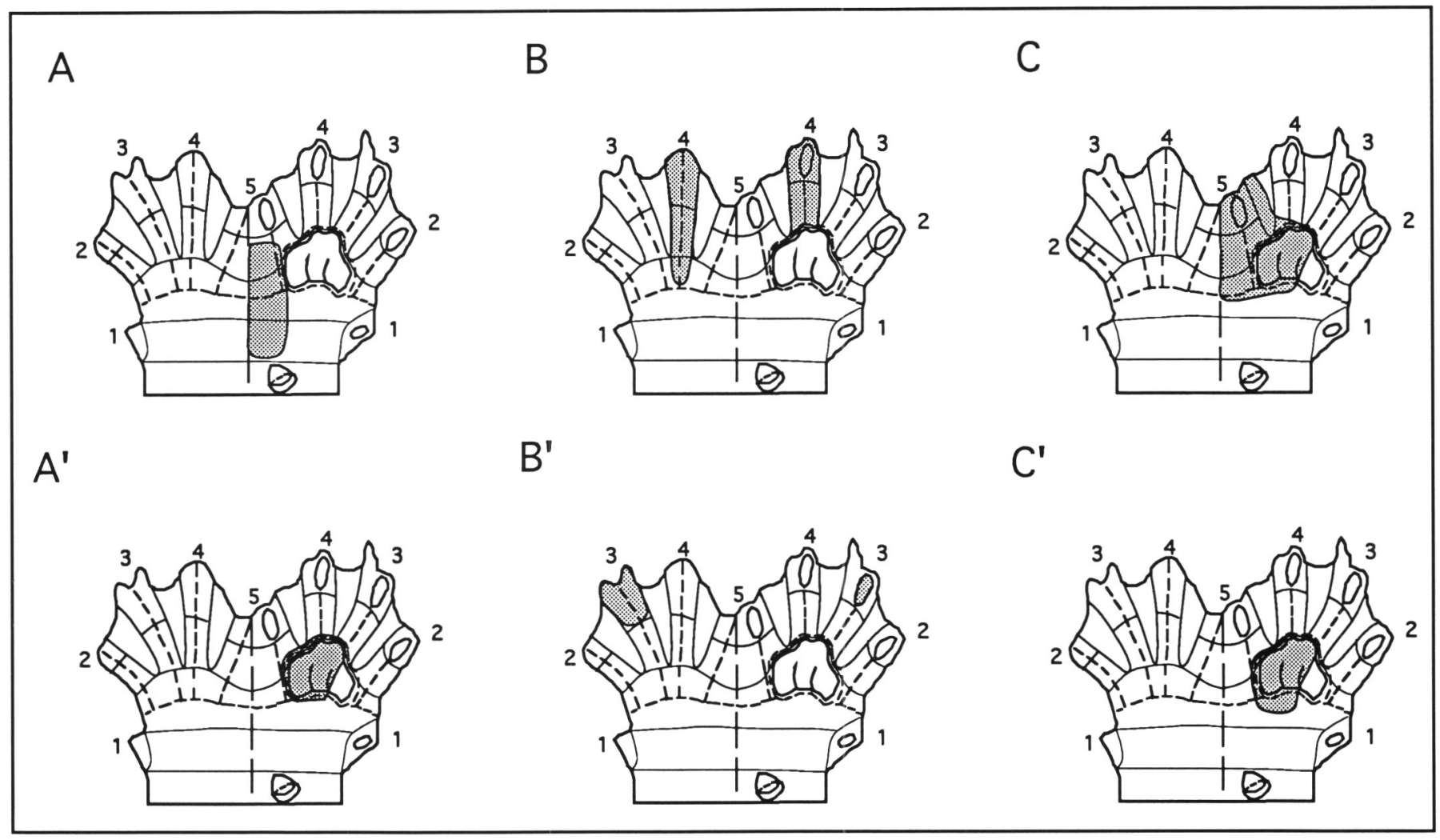

Figure 7. Reconstruction of receptive fields for sites indicated by large open circles in the ulnar nerve zone and combined nerve zone illustrated in Figure $6 A$ and $A$ ' before (upper) and after (lower) ulnar nerve transection. A and A'. Receptive field recorded in the ulnar nerve zone. $B$ and $B$ '. Receptive field recorded in combined nerve zone. $C$ and $C^{\prime}$. Receptive field recorded in ulnar nerve zone and combined nerve zone illustrating the loss of input from autonomous skin zone but not from the overlap skin zone after ulnar nerve cut. 
Figure 8 illustrates the results of the second remap after nerve cut and compares the first remap (beginning immediately after nerve cut) with the later remap (beginning $42 \mathrm{hr}$ after nerve cut) using symbols introduced in the previous figure. The main finding is that the cortex previously served by the ulnar nerve remains, in large part, unresponsive to peripheral stimulation up to as many as $\mathbf{5 0}$ hours after nerve cut. However, a few new receptive fields appeared in both the ulnar nerve zone and the combined nerve zone of some cases. One example of a new receptive field in the ulnar nerve zone at a site unresponsive during the first remap is shown in Figure 9A, A'. During the original mapping, neurons in this site were responsive to stimulation of the ulnar side of the MP and the pad of D5. During the second remapping, neurons at this site were responsive once again, but this time the new receptive field included part of the metacarpal pad and the hair lying along the sides of the pad.
Neurons at other sites became unresponsive at locations that were active during the first remapping. An example is shown in Figure 9B, B'. Prior to nerve cut, neurons at this location were responsive to stimulation of the distal part of the ventral side of D4. After nerve cut, the receptive field expanded to include the ventral side of D3 and the dorsal distal segment of D4. However, during the second remapping this site was unresponsive. Figure $8 \mathrm{~A}$ shows a new response was recorded in the ulnar nerve zone as indicated by the asterisk, but during the remapping shown in Figure 8A' that site was unresponsive. Note that within the ulnar nerve zone most sites were still unresponsive to peripheral stimulation; however, three of the sites had acquired new receptive fields. In the other cases illustrated in Figure 8, the ulnar nerve zone remained largely unresponsive to stimulation and did not change between the first and second remapping.

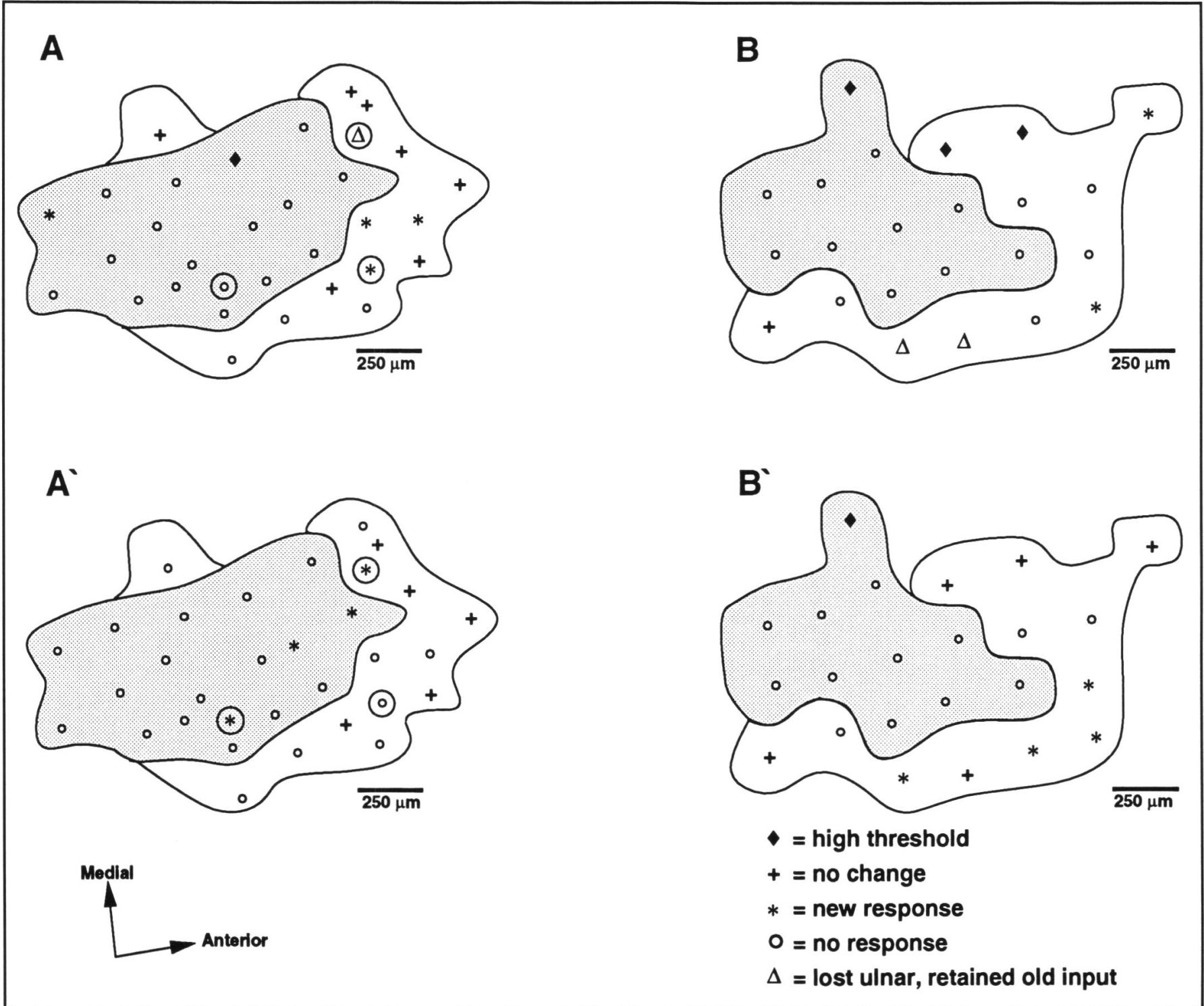

Figure 8. Reconstruction of receptive fields during first remapping following nerve cut (upper) and second remap (lower) in two cats. Upper traces are identical to cases illustrated in 6A,C. The same symbols are used in the lower trace to indicate changes in receptive fields relative to the first remap in the upper trace. Large circles in A indicate sites where receptive fields are reconstructed in Figure 9. 
In the combined nerve zone, changes in receptive fields were encountered more commonly at the second remapping. There were some sites that had been responsive at the first remapping after transection but lost the response before the second remapping was made. There were sites that had no response which became responsive and there were sites that were responsive to high threshold tap stimulation which acquired a cutaneous receptive field by the second remapping. These changes can be seen by the differences in the symbols at each site in the combined nerve zones of the upper and lower parts of Figure 8. An example of a site that lost its ulnar nerve input during the first remapping, but responded to stimulation of the neighboring digit on the second remapping is illustrated in Figure 9C, C'. This site was responsive to stimulation of both the ventral pad of D5 and the MP. Following nerve cut, the D5 component was lost, but stimulation of the MP continued to evoke a cortical response. Following the second remapping, the MP component was also lost, and the site became responsive to stimulation of entire dorsal part of D3, parts of D3 and D2 as well as the pad of D3. Thus the map could be expected to change later in the mapping process.

\section{Multiple Mapping: Sham-Operated Control}

Our results suggest that immediately following nerve transection a large portion of previously innervated cortex is subsequently unresponsive to peripheral input and remains so at least up to two days after nerve cut. Since multiple electrode penetrations were made into nearly identical cortical sites, it was possi- ble that unresponsive cortical sites resulted from tissue damage caused from the electrode. Furthermore, to the best of our knowledge, sham-control animals have not been incorporated in previous studies of cortical reorganization, and the possibility cannot be ruled out that new receptive fields are a consequence of a continually changing cortical map.

These concerns prompted the examination of multiple mapping on one sham-operated control animal. All surgical and recording procedures were identical to those previously described except the skin proximal to the elbow was slit and the ulnar nerve was exposed but not transected as in the experimental animals. These results are illustrated in Figure 10. The original map of the territory explored is shown in Figure 10A; the ulnar nerve zone of cortex receiving input from the ulnar nerve is outlined with the dark stippling and the combined nerve zone of cortex receiving input from the ulnar nerve in combination with the median and/or radial nerves is highlighted with the lighter stippling. Each site, illustrated in Figure 10A, where an electrode penetration was made is indicated by a closed circle (responsive site) or open circle (unresponsive site). Examination of Figure $10 \mathrm{~A}$ reveals that the overall map organization is not unlike the topographically organized maps illustrated in Figures 3-5.

Immediately following the completion of the original map, the cortex was remapped (first remap); the results of this immediate remapping are illustrated in Figure 10B. The data from the first remap were generated in relationship to the data from the
A

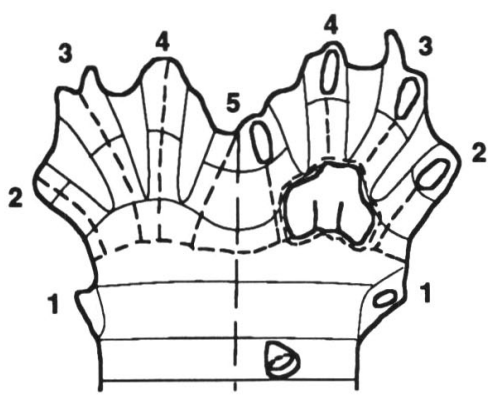

$A^{\prime}$

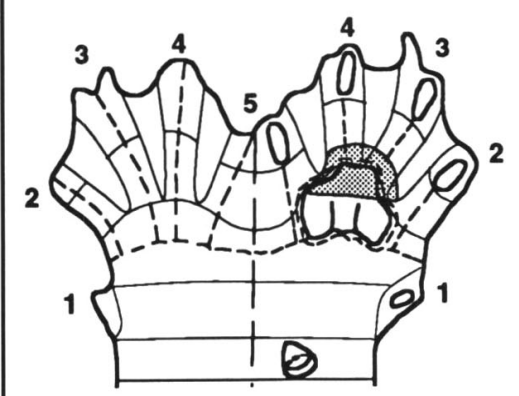

B

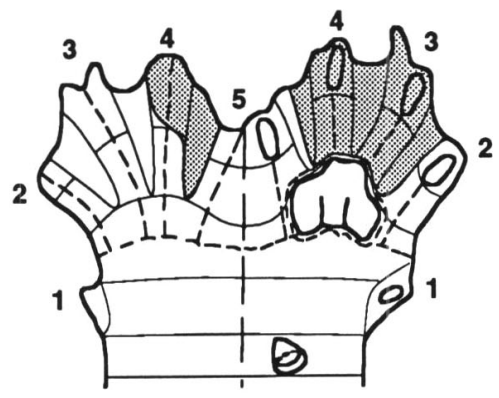

$\mathbf{B}^{\prime}$

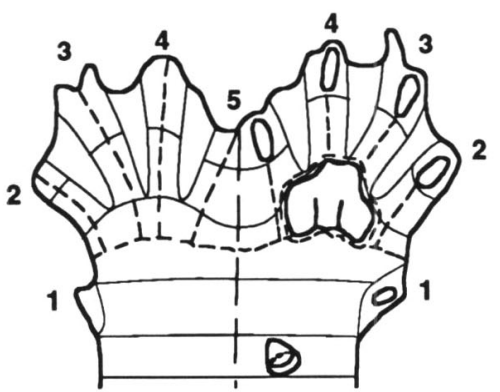

C

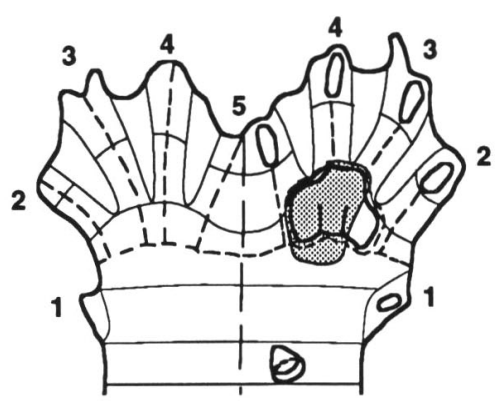

$C^{\prime}$

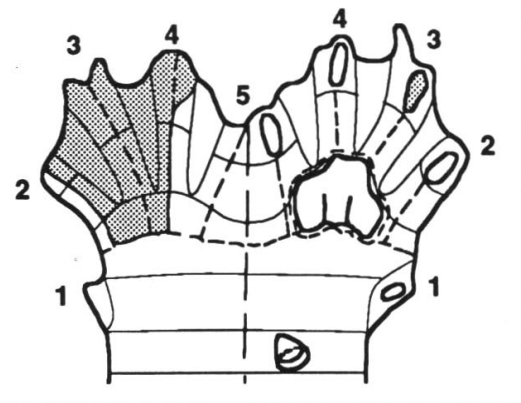

Figure 9. Receptive fields recorded during the first remap (upper) and second remap (lower) for the cases illustrated in Figure 8A, B (large circles). A. Shows an unresponsive site in the uinar nerve zone that acquired a new receptive field during the second remapping (lower). $B$. lllustrates a receptive field in the combined nerve zone that was lost during the second remap. C. Shows a new response obtained in the combined zone during the second remapping. 
original map. Receptive fields were divided into two categories: recording sites where no change in receptive field was observed between the two maps (crosses), and recording sites where a slight change in receptive field was observed between the two maps (open square). Other categories such as no response, high threshold or new receptive field were not included since no instances of the occurrence of these response types were encountered. Inspection of Figure 10B reveals that in contrast to the map obtained immediately following nerve transection, no new unresponsive sites were observed in the immediately remapped cortex of the control cat. Instead, all sites in ulnar nerve zone cortex and combined nerve zone cortex were responsive to peripheral input. The majority of these sites had receptive fields that exhibited little change from the receptive field recorded in the original map. In a limited number of cases, sites were found (open square) that were responsive to input from a portion of the same skin location as observed in the original map, but the receptive field recorded during first remapping was slightly increased or decreased in size, However, in all cases, a portion of the original receptive field was preserved within the modified receptive field. These modified receptive fields were not limited to the ulnar nerve innervated cortex, but appeared throughout all regions of cortex examined. From the total number of electrode penetrations, approximately $12 \%$ of the receptive fields recorded in the first map were modified compared to the original map. However, close inspection of the ulnar nerve zone and the combined nerve zone indicates that twice as many modified response sites were encountered in the combined nerve zone $(37 \%)$ as compared to the ulnar nerve zone $(18 \%)$ where the receptive field was slightly altered in size. Whether these modified receptive fields reflect actual differences in the two zones is unclear at this time.

The cortex was then remapped (second remap) a second time $20 \mathrm{hr}$ after the conclusion of the first remap and the results are illustrated in Figure 10C. The results of the second remapping are compared to the results of the immediately remapped cortex (second map). Once again, the majority of sites had receptive fields identical to those observed in immediate remapped cortex. In the majority of the modified sites in the second remap, the receptive fields were nearly identical to the receptive fields in the original map. All sites that were responsive in the original and first remapped cortices were also responsive $20 \mathrm{hr}$ later. Thus, multiple electrode penetrations into identical or nearly identical cortical sites in a sham-operated control cat are not sufficient to produce unresponsive cortex. However, whether the few slightly modified receptive fields result from an error in electrode placement or from a continually changing cortex remains to be determined.

\section{Receptive Field Mapping Under Ketamine}

Since many cortical sites remained unresponsive immediately following nerve cut, and other investigators ${ }^{9,16-19}$ have reported immediate reorganization in a variety of species using Ketamine, we examined two additional animals using the same experimental protocol as described for the nerve cut with the exception that Ketamine was used as the anesthetic. An example of the map of one of these animals is illustrated in Figure 11. The original map before nerve cut is shown at the upper left of the figure in terms of ulnar nerve zone (shaded) and combined nerve zones. The same lettering and symbols are used in this figure as previously described. The area of the combined nerve zone appears larger than the area of the ulnar nerve zone; however, greater variability in size and shape would be expected among individuals if more animals had been included in the sample. Nonetheless, several important observations were still seen in the map organization immediately after nerve cut and were similar to observations made in animals studied under Nembutal.

The lower part of Figure 11 illustrates the result of first remapping immediately following nerve cut using the previously described symbols. The first important observation is that immediately after nerve cut many unresponsive sites are seen within the ulnar nerve zone. However, many more responsive sites were found in the ulnar nerve zone than was the case when the mapping was accomplished under Nembutal anesthesia. Many of these sites were activated by high-threshold tapping, but new responses were also observed. At four sites new responses were found following stimulation of neighboring regions of the skin. When new responses were observed, they were generally found at locations in the ulnar nerve zone bordering on the combined nerve zone rather than sites located in the middle of the ulnar nerve zone. An example of a new receptive field in the ulnar nerve zone is illustrated in the paw map shown at the right of the Figure 11A and $A^{\prime}$. The original receptive field (upper right) for that site is restricted to the dorsal side of the fifth digit. Following nerve cut, stimulation of D5 no longer evoked a cortical response; instead, this cortical site was driven by stimulation from the adjacent dorsal side of $\mathrm{D} 4$, web between D3 and D4, and the ulnar side of the dorsal aspect of D3. Although too few to test statistically, the number of new receptive fields within the ulnar nerve zone was greater than in any of the cases studied under Nembutal. Conversely, the proportion of unresponsive sites was also reduced in cats studied under Ketamine compared to animals mapped under Nembutal.

Although the combined nerve zone was large in the original map, most of these sites showed no change in response following nerve cut. Many of the sites that showed change were located immediately adjacent to the ulnar nerve zone. Other locations within the combined nerve zone that showed a change lost their ulnar nerve contribution, but retained the contribution from neighboring nerves. An example of one of these sites is shown in the right of Figure 11B and B'. The receptive field before nerve cut included the dorsal part of D4 and D5 and the interconnecting web. After nerve cut, the D5 contribution was lost as well as the input from the radial side of D4. Neurons at that site were now only responsive to input from the ulnar side of D4 and the adjacent web. Thus it was not always easy to predict the effect of nerve cut on the receptive field of sites in the combined nerve zone.

\section{Discussion}

The details of the organization of the primary somatosensory cortex served by the ulnar nerve were studied before and immediately after ulnar nerve transection in the cat. As part of the study we adopted procedures to reduce many possible sources of experimental error. These procedures included (i) selecting the ulnar nerve representation to study since it lies beneath the postsigmoid gyrus rather than the median or radial nerve representations that lie, in part, along the bank of the coronal sulcus, (ii) maintaining the animal's head in the same fixed position throughout all mapping and remapping to maintain an invariant angle of electrode placement in the brain, (iii) selecting one recording depth to map receptive fields before and after nerve cut, (iv) using the same experimenter to map receptive fields so 


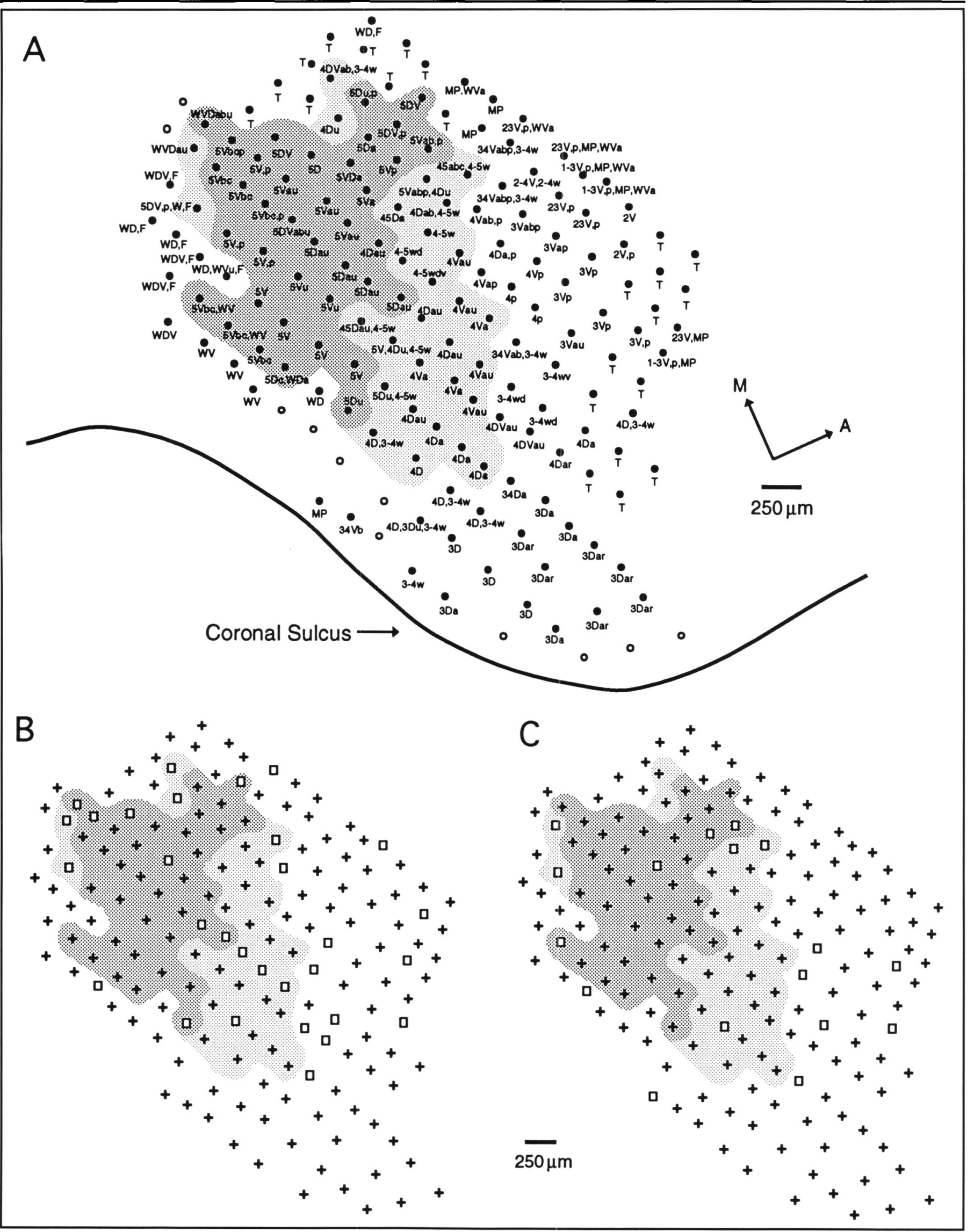

Figure 10. Reconstruction of multiple mapping in the sham-operated control. A. Original map of the ulnar nerve innervated somatosensory cortex. The ulnar nerve zone is indicated by dark stipple and the combined nerve zone is indicated by lighter stipple. Recording sites in upper figure are indicated by black dots and receptive fields recorded at each site are indicated by the previously described nomenclature. B. Second map immediately after the original map. C. Third map $20 \mathrm{hr}$ after the original map. Open circle $=$ no response; crosses $=$ no change in receptive field; open square $=$ modified receptive field. 


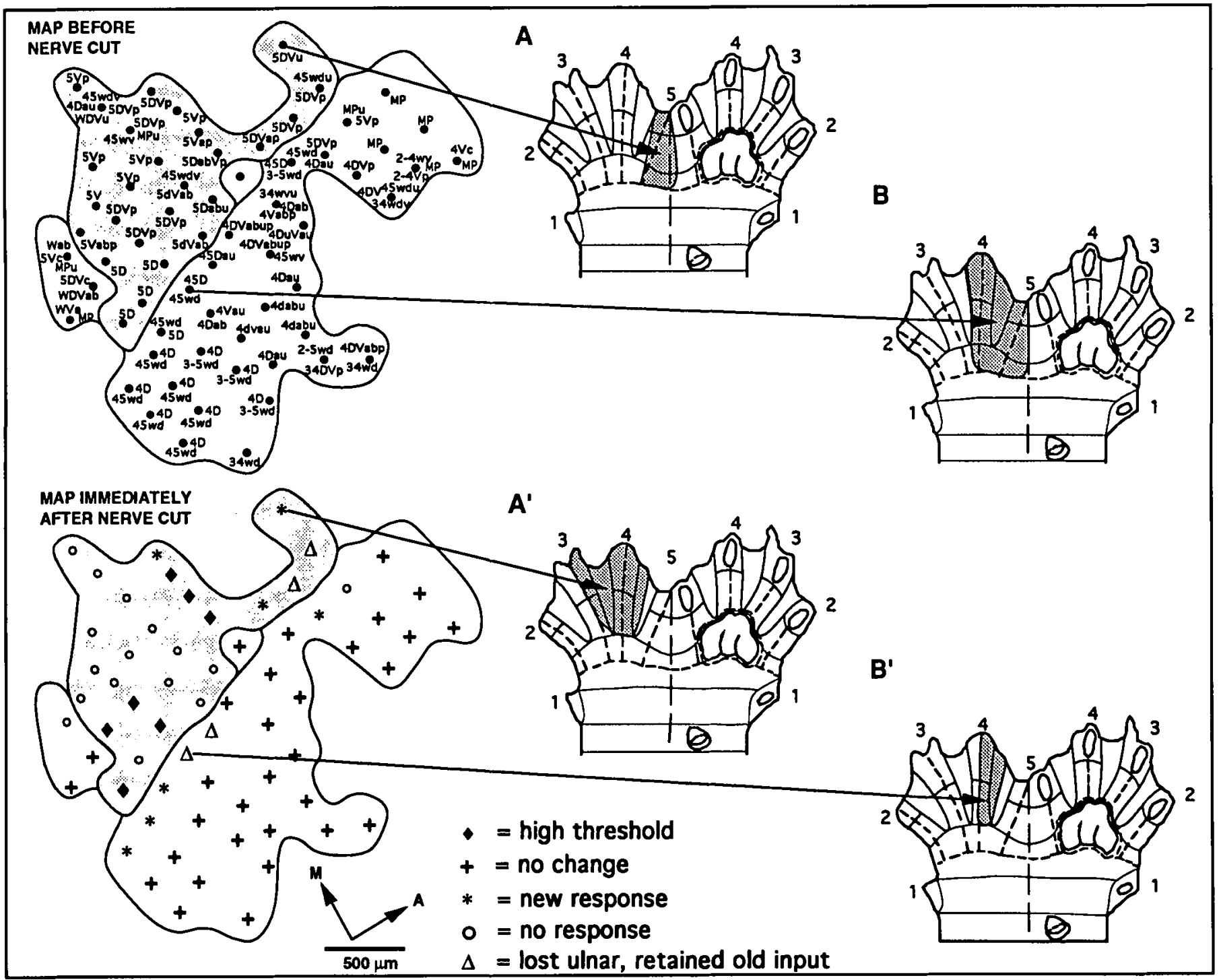

Figure 11. Reconstruction of the map obtained before (upper left) and immediately after (lower left) nerve cut for an animal anesthetized with Ketamine along with the receptive field recorded before (upper figurines) and after nerve cut (lower figurines). Recording sites in upper figure are indicated by black dots and receptive fields recorded at each site are indicated by the previously described nomenclature. Receptive field nomenclature is as previously described.

that differences in mapping technique would be controlled and, (v) mapping before and after nerve cut in the same animal. By employing these measures along with high quality photographs of the cortical surface to mark the location where electrode placements were made, we were able to control many variables that might otherwise bias the results. We also included a shamoperated animal to control for the possibility that the large number of unresponsive sites might result from damage to cortex from multiple electrode penetrations or, in those cases where cortical reorganization was observed, that the new receptive fields were a consequence of deafferentation rather than the result of cortical neurons constantly undergoing changes in their receptive fields. However in our estimation one of the most important factors that must be taken into account in studies of reorganization is the individual differences observed in the size and shape of the ulnar nerve representation in the primary somatosensory cortex. Individual differences in sulcal patterns have been reported in $\mathrm{cat}^{22}$ as well as individual variability in the representation of the hand surface in monkeys. ${ }^{23}$ Differences in this factor can be minimized if the same animal is mapped before and after nerve cut so that alterations in the cortical map are not mistaken for reorganization but rather seen as map variability.

Unlike the variability of the peripheral nerve representation observed in primary somatosensory cortex, the distribution of the ulnar nerve in the cat forepaw is more consistent between animals. The pattern of peripheral nerve innervation of the cat forepaw has been described. ${ }^{21,24}$ The ulnar nerve innervates the entire fifth digit and ulnar side of the wrist, the ulnar side of the web between digits 4 and 5 and the carpal pad. Rather than having a sharp boundary between the ulnar innervated skin and neighboring skin innervated by the median and radial nerves, a transition zone exists which receives overlapping input from both the ulnar and the median nerves on the ventral side and 
from the ulnar and the radial nerves on the dorsal side of the paw. The result is a rather large and relatively constant ulnar nerve autonomous zone and a slightly more variable overlap zone receiving overlapping input from two peripheral nerves.

In contrast to the stable pattern in the periphery, the representation of the ulnar nerve in the ulnar nerve zone and combined nerve zone in primary somatosensory cortex varies in size and shape among individuals. Nevertheless there are certain consistencies: (i) the autonomous ulnar nerve representation and the overlapping nerve representation are found on the posterolateral side of the body map lying along the postsigmoid gyrus adjacent to the coronal sulcus; (ii) the ulnar nerve zone is organized internally, having a somatotopic organization that progresses from the ventral paw receptive fields located lateral to the more medially located dorsal paw receptive fields, although reversals in this progression are also seen; (iii) the representations of the forearm and wrist are found on the posterolateral side of the map; and (iv) the fourth digit representation and part of the third digit representation lie anterior to representation of the fifth digit.

After transection of the ulnar nerve, the expectation derived from the published data had been that a large number of sites in primary somatosensory cortex should begin to respond to novel inputs as reported in squirrel monkey, ${ }^{9}$ raccoon, ${ }^{12-15}$ bat, ${ }^{16,17}$ and rat. ${ }^{18,19}$ Instead, only a very few recording sites were activated by low-threshold stimuli and a few more were activated by rather intense somatic stimuli. Most of the area lacked responses. The new receptive fields found within the ulnar nerve zone were activated by low-threshold cutaneous stimuli and seemed relatively normal in size and response characteristics.

Other investigators have also reported silent zones in primary somatosensory cortex immediately following nerve cut. For example, Merzenich and colleagues ${ }^{9}$ failed to record new receptive fields in the cortex in the owl monkey immediately after median nerve cut, although they did observe new receptive fields in the median nerve territory of the squirrel monkey as well as a large unresponsive region immediately after deafferentation. A similar absence of excitatory responses was also reported in raccoon after digit amputation; ${ }^{12}$ however, these investigators did report a high percentage of "Off" responses and suppression responses from the neighboring digits in denervated cortex which they interpreted as new cortical responses. Conversely, Metzler and Marks ${ }^{6}$ used lidocaine $\mathrm{HCl}$ as an epidural block to remove input from L4-L5 dorsal roots and reported that approximately $28 \%$ of their cells had new receptive fields. This percentage was much higher than the percentage of new cutaneous receptive fields $(9 \%)$ we found.

The sparseness of new receptive fields within the pure ulnar nerve zone after nerve cut in our experiments (with the exception of complete unresponsive cortex in the denervated zone in owl monkey reported by Merzenich et al. ${ }^{9}$ ) provoked speculation that the barbiturate anesthesia might have been responsible, in part, for these results that differed so greatly from those previously reported in primate and bat. However, changing to Ketamine, the anesthetic used by Calford and Tweedale, ${ }^{16,17}$ Wall and Cusick, ${ }^{18,19}$ and Merzenich et al. ${ }^{9}$ did not resolve the discrepancy. Cats examined under Ketamine anesthesia had a few more novel receptive fields following ulnar nerve transection than when Nembutal had been used, but the majority of recording sites were unresponsive to somatic stimuli. Still several sites were found that could be driven by high-threshold stimulation. Unlike immediate reorganization reported in squirrel monkey (Merzenich et al., ${ }^{9}$ see their Figure 2) where large silent zones were reported to occur both at the center and on the fringe of the denervated cortex, our novel responses appeared entirely at the edges of the ulnar nerve zone leaving the interior of the region served by the ulnar nerve completely devoid of responses. Within the ulnar nerve zone most new responses were observed primarily at the boundary.

The experiments with Ketamine suggest that our results are not entirely due to the anesthesia, for we obtained a much greater proportion of unresponsive sites or sites that could be activated only by tap stimulation than that obtained by Merzenich et al. ${ }^{9}$ and Calford and Tweedale et al..$^{16,17}$ using the same anesthetic. In other reports of immediate reorganization of raccoon ${ }^{13,14}$ and cat, ${ }^{6}$ animals were studied under inhalation anesthesia, which may produce less cortical suppression than Nembutal, and these reports show a large number of unresponsive sites.

No investigators studying reorganization have mapped the somatosensory cortex in terms of ulnar nerve and combined nerve zones, although many of these previous studies involved denervation of a peripheral nerve, so there is no direct way of comparing our maps with those of others. Recordings in the combined nerve zone following nerve cut produced results that were not easily predictable prior to nerve cut since these regions receive, in part, input from neighboring intact nerves. For example, unresponsive sites were found in the combined nerve zone even though a portion of their innervation remained from the neighboring nerve after nerve cut. Furthermore, new receptive fields were occasionally found in the combined nerve zone. These findings suggest, as Merzenich et al. ${ }^{23}$ have pointed out, that the somatosensory cortex is not static, but rather a dynamic system where shifts in receptive fields may be common within limits. These unpredicted changes in the receptive field may reflect this ongoing dynamic process.

The results of the sham-operated control possibly address this point by supporting the notion that receptive fields may be constantly changing over time, but that the changing receptive fields are anchored around a centralized region of skin. However, it is also tenable that our modified receptive fields may reflect a slight deviation in the replacement of the recording electrode in the subsequent maps that may lead to a slight alteration in the receptive field. Interestingly enough, the modified fields always retain a large part of the original receptive field and in that way are different from the reorganized cortex resulting from nerve transection. At present neither explanation of a constantly changing cortex or error in electrode replacement can be discounted.

Our results suggest that although large unresponsive areas remain in the deafferented cortex, nonetheless new receptive fields are occasionally found and that slight differences may exist which are dependent on the anesthetic used during mapping. That these few uncovered responses were not apparent in the recordings prior to nerve transection implies that there must have been sudden changes in the relative effectiveness of some existing connections. On the basis of observation from earlier work, we believe that the changed effectiveness of excitatory drive after deafferentation is due to a reduction of intracortical 
inhibition provoked by the deafferentation. Hicks ${ }^{25}$ and Dykes ${ }^{26}$ showed that there was an unexpressed excitatory fringe to the majority of receptive fields of the cortical neurons. The expression of this fringe (which often had an area greater than the excitatory center) was controlled by GABAergic inhibition. These suppressed inputs provide an existing reservoir of afferent connections for such a mechanism. This observation, combined with the fact that Nembutal anesthetic enhances the binding between GABA and its receptor (for review, see Hicks et al. ${ }^{27}$ ) thereby reducing responses of cortical cells to peripheral stimulation, accounts for the smaller number of new receptive fields with Nembutal than with Ketamine.

To account for the reorganization observed in this study under both Nembutal and Ketamine anesthesia, what must be hypothesized is that transection of a peripheral nerve produces a decrease in intracortical inhibition. Indirect evidence for such a phenomenon was provided by Dykes and Lamour ${ }^{28}$ and Lamour and Dykes ${ }^{29}$ when they showed that following nerve transection, neurons in the rat hindlimb somatosensory cortex behave as though they were released from inhibition, being more frequently spontaneously active at higher rates than in normal cortex and giving exaggerated responses to glutamate. The cellular mechanisms linking peripheral nerve transection to this release from inhibition are unknown. One possibility is that normally in a peripheral nerve there is an afferent barrage constantly driving cortical neurons. Such signals have never been demonstrated, but the fact that important changes in the organization of cortical somatosensory receptive fields follow transient nerve blocks ${ }^{6}$ makes the existence of such activity a tenable hypothesis.

\section{ACKNOWLEDGEMENTS}

The authors are grateful for the suggestions made by Drs. T.P. Hicks and D.D. Rasmusson. We thank M. Waters for editing. This work was supported by NSF grant BNS-88-02766 to R.S.W. C.X.L. is a visiting scientist from China Medical University. This work is dedicated to the memory of my mentor, Leslie D. McIntosh.

\section{REFERENCES}

1. Dykes RW. Parallel processing of somatosensory information: a theory. Brain Res Rev 1983; 6: 47-115.

2. Kaas JH, Nelson RJ, Sur M, Lin C-S and Merzenich MM. Multiple representations of the body within the primary somatosensory cortex of primates. Science 1979; 204: 521-523.

3. Chapin JK, Lin CS. Mapping the body representation in the SI cortex of anesthetized and awake rats. J Comp Neurol 1984; 229: 199-213.

4. Welker C. Microelectrode delineation of fine grain somatotopic organization of $\mathrm{Sm} 1$ cerebral neocortex in albino rat. Brain Res 197I; 26: 259-275.

5. Kalaska J, Pomeranz B. Chronic paw denervation causes an agedependent appearance of novel responses from forearm in "paw cortex" of kittens and adult cats. J Neurophysiol 1979; 42: 618633.

6. Franck JI. Functional reorganization of cat somatic sensory-motor cortex $(\mathrm{Sml})$ after selective dorsal root rhizotomies. Brain Res 1980; 186: 458-462.

7. Metzler J, Marks PS. Functional changes in cat somatic sensorymotor cortex during short-term reversible epidural blocks. Brain Res 1979; 177: 379-383.

8. Merzenich MM, Kaas JH, Wall J, et al. Topographic reorganization of somatosensory cortical areas $3 \mathrm{~b}$ and 1 in adult monkeys following restricted deafferentation. Neuroscience 1983; 8: 33-55.
9. Merzenich MM, Kaas JH, Wall JT, et al. Progression of change following median nerve section in the cortical representation of the hand in areas $3 \mathrm{~b}$ and $\mathbf{l}$ in adult owl and squirrel monkeys. Neuroscience 1983; 10: 639-665.

10. Merzenich MM, Kaas JH. Reorganization of mammalian somatosensory cortex following peripheral nerve injury Trends Neurosci 1982; 5: 434-436.

11. Pons TP, Garraghty PE, Ommaya AK, et al. Massive cortical reorganization after sensory deafferentation in adult macaques. Science 1991; 252: 1875-1860.

12. Rasmusson DD, Turnbull BG. Immediate effects of digit amputation on SI cortex in the raccoon: unmasking of inhibitory fields. Brain Res 1983; 288: 368-370.

13. Turnbull BG, Rasmusson DD. Acute effects of total or partial digit denervation on raccoon somatosensory cortex. Somatosen Motor Res 1990; 7: 365-389.

14. Kelahan AM, Doetsch GS. Time-dependent changes in the functional organization of somatosensory cerebral cortex following digit amputation in adult raccoons. Somatosen Res 1984; 2 : 4981 .

15. Kelehan AM, Ray RH, Carlson LV, Massey CE, Doetsch GS Functional reorganization of adult raccoon somatosensory cerebral cortex following neonatal digit amputation. Brain Res 1981; 223: 152-159.

16. Calford MB, Tweedale $R$. Immediate and chronic changes in responses of somatosensory cortex in adult flying-fox after digit amputation. Nature 1988; 332: 446-448.

17. Calford MB, Tweedale $R$. The capacity for reorganization in adult somatosensory cortex. In: Rowe M, Aitkin L, eds. Information Processing in Mammalian Auditory and Tactile Systems. New York: Alan R. Liss, Inc., 1990: 221-236.

18. Wall JT, Cusick CG. Cutaneous responsiveness in primary somatosensory (S-I) hindpaw cortex before and after partial hindpaw deafferentation in adult rats. J Neurosci 1984; 4: 1499 1515.

19. Wall JT, Whiting JH Jr, Wiley RG. Temporal progression of cortical reorganization following nerve injury. Brain Res 1991; 537: 355-358.

20. Armstrong-James M, Millar JJ. Carbon fiber microelectrodes. Neurosci Meth 1979; 1: 279-287.

21. Waters RS, Li CX, Oladehin A, Johnson EF, Dykes RW. Ulnar nerve innervation of paw and SI contex of cat: substrate for reorganization. Neuroreport 1990; 1: 225-229.

22. Kawamura K. Variations of the cerebral sulci in the cat. Acta Anat 1971; 80: 204-221.

23. Merzenich MM, Nelson RJ, Kaas JH, et al. Variability in hand surface representations in areas $3 \mathrm{~b}$ and 1 in adult owl and squirrel monkeys. J Comp Neurol 1987; 258: 281-296.

24. Kitchell RL, Canton DD, Johnson RD, Maxwell SA. Electrophysiologic studies of cutaneous nerves of the forelimb of the cat. J Comp Neurol 1982; 210: 400-410.

25. Hicks TP, Dykes RW. Receptive field size for certain neurons in primary somatosensory cortex is determined by GABA-mediated intracortical inhibition. Brain Res 1983; 274: 160-164.

26. Dykes RW, Landry P, Metherate R, Hicks TP. Functional role of GABA in cat primary somatosensory cortex: shaping receptive fields of cortical neurons. J Neurophysiol 1984; 52: 1066-1093.

27. Hicks TP, Landry P, Metherate R, Dykes RW. Functional properties of neurons mediated by GABA in cat somatosensory cortex under barbiturate and urethane anesthesia. In: Rowe MJ, Willis WD, eds. Development, Organization, and Processing in Somatosensory Pathways. New York: AR Liss Inc., 1985: 265276.

28. Dykes RW, Lamour Y. An electrophysiological laminar analysis of single somatosensory neurons in partially deafferented rat hindlimb granular cortex subsequent to transection of the sciatic nerve. Brain Res 1988; 449: 1-17.

29. Lamour Y, Dykes RW. Somatosensory neurons in partially deafferented rat hindlimb granular cortex subsequent to transection of the sciatic nerve: effects of glutamate and acetylcholine. Brain Res 1988; 449: 18-33. 